\title{
Load Balancing Strategy using Round Robin Algorithm
}

\author{
Dong Hyun Youm1), Ravi Yadav2)
}

\begin{abstract}
Current cloud computing environment serves in practically every field of our life. Be that as it may, while satisfying parts and loads of client solicitations it confronts couple of confinements to be overcome. Alongside giving us offices like virtualization, asset sharing, universality, utility registering it request that we concentrate on issues like security, authentication, and adaptation to internal failure, load balancing, and availability. Part of work is being completed to accomplish stack adjusting in cloud framework now days. There are such a large number of load adjusting calculations playing out their errand on various layers of cloud with various levels of complexities. To accomplish incredible level of load adjusting individuals utilize numerous mind boggling calculations. Be that as it may, thus it includes all the more handling burden the framework executing such logic.
\end{abstract}

Keywords : cloud computing, virtualization, load balancing, security, round robin, security.

\section{Introduction}

Actualizing load adjusting in your application just appropriating the approaching solicitations stack among the accessible demand executing hubs[1-3]. Stack adjusting deals with the executing hubs that they don't get over-burden with the client ask. So that by utilizing some planning calculations the load is dispersed among servers[4-6]. Each server is having a record state, as accessible, occupied, and sit still. At the point when there is an approaching solicitation these planning calculation checks whether the server is sit or not. As indicated by that the load is disseminated to the servers.

Basically there are 2 types of load balancing algorithm depending on their implementation method according to:

A. Static Load Balancing Algorithm: It does not depend on the current state of the system. Earlier before theincoming request it decides that at which server the request will be executed.

Received(June 13, 2016), Review Result(1st: June 30, 2016, 2015, 2nd: July 25, 2016), Accepted(September 10, 2016)

${ }^{1}$ Department of Applied Computer Engineering, Dankook University, 152 Jukjeon-ro, Suji-gu, Gyeonggi-do email: daivid@gmail.com

${ }^{2}$ (Corresponding Author) Department of Computer Science and Engineering, KL University

email: ravibyadav@gmail.com 
B. Dynamic Load Balancing Algorithm: The load balancer analyses the current load statistics at each available server and executes request at appropriate server.

In the present cloud foundation the load adjusting idea at few levels. It can be actualize by load adjusting at Service Broker, Server group and the Virtual Machine Monitor (VMM), have demonstrated an essential cloud foundation. Area $\mathrm{N}$ demonstrates geographic area of the clients. Client bases are gathering of clients sending solicitations to the server. With the assistance of Service Broker client demand will distinguish the appropriate server which can satisfy their solicitations. Here we have indicated VM stack balancer at executing hub bunch. In more terrible circumstances VM stack balancer takes choice to relocate VM instead of VM load with help of VMM[7-9]

In essential round robin calculation the Load balancer allots a VM to asking for hub in cyclic way similarly among every single accessible hub. Fundamental preferred standpoint of this calculation is that it uses every one of the assets in an adjusted request and equivalent quantities of VMs are apportioned to every one of the hubs.

Stack adjusting calculation, actualized at any level of cloud, must attempt to satisfy any or the majority of the accompanying qualities as indicated by :

a)Maximum context switches, CPU utilization, throughput and

b) Minimum Turnaround time, Waiting time, Response time.

With context switches we mean exchanging among the CPU states put away before so execution can be halted from it. Throughput demonstrates the quantity of procedures finished per unit time. Usage of CPU is determined by CPU use, it ought to be as high as could reasonably be expected. Turnaround time is total of the time a procedure spend in prepared line sitting tight for memory alongside doing enter yield procedures and executing on CPU. Holding up time is the measure of time a procedure holds up in prepared line. Reaction time is the time the framework begins reacting the demand, not the time it finishes reacting.For the task scheduling based on RR, throttled, min-min, min-max the criteria include the following:

Context Switch: A setting switch is figuring procedure of putting away and reestablishing condition of a CPU with the goal that execution can be continued from same point at a later time. Setting switch are generally computationally serious, prompt to wastage of time, memory, 
scheduler overhead such an extensive amount the plan of working framework is to streamline these switches.

Throughput: Throughput is characterized as number of process finished per unit time. Throughput will be moderate in round robin planning execution. Setting switch and throughput are relative to each other.

CPU Utilization: We need to keep the CPU as occupied as would be prudent.

Turnaround Time: Turnaround time is total of periods spent holding up to get into memory, holding up in prepared line, executing on CPU and doing include yield. It ought to be less.

Waiting Time: Waiting time is the measure of time a procedure has been holding up in prepared line. The CPU booking calculation does not influence the measure of time amid which a procedure executes or inputs yield; it influences just the measure of time that a procedure spends holding up in prepared line.

Response Time: Response time is the time it takes to begin reacting, not the time it takes to yield the reaction. Substantial reaction time is a disadvantage in round robin design as it prompts to debasement of framework execution.

A good scheduling algorithm must possess following Characteristics:

1. Minimum context switches.

2. Maximum CPU utilization.

3. Maximum throughput.

4. Minimum turnaround time.

5. Minimum waiting time.

6. Minimum response time.

The random arrival of load in such an environment can cause some server to be heavily loaded while other server is idle or only lightly loaded. Equally load distributing improves performance by transferring load from heavily loaded server. Efficient scheduling and resource allocation is a critical characteristic of cloud computing based on which the performance of the system is estimated. The considered characteristics have an impact on cost optimization, which can be obtained by improved response time and processing time.

\section{Proposed System}




\subsection{Existing Algorithms}

\subsubsection{Round Robin Algorithm}

The round-robin (RR) planning calculation is composed particularly for time-sharing systems. It is like FCFS planning, yet pre-emption is further to switch between procedures.

A little unit of time, called a time quantum or time cut, is distinctive. The prepared line is considered as a roundabout line. To perform RR booking, we keep the prepared line as a FIFO line of procedures. New procedures are added beside the prepared line. The CPU scheduler picks the primary procedure from the prepared line, plans a time quantum to hinder after some time, and dispatches the procedure. The procedure may have a CPU burst not exactly the time quantum. For this situation, the procedure itself will release the CPU willfully. The scheduler will then continue to the following procedure in the prepared line. Something else, if the CPU burst of the at present running procedure is longer than the given time quantum, the time will go off and will bring about a hinder to the OS.

A context switch will be executed, and the procedure will be put at the tail of the prepared line. The CPU scheduler will then choose the following procedure in the readied line.

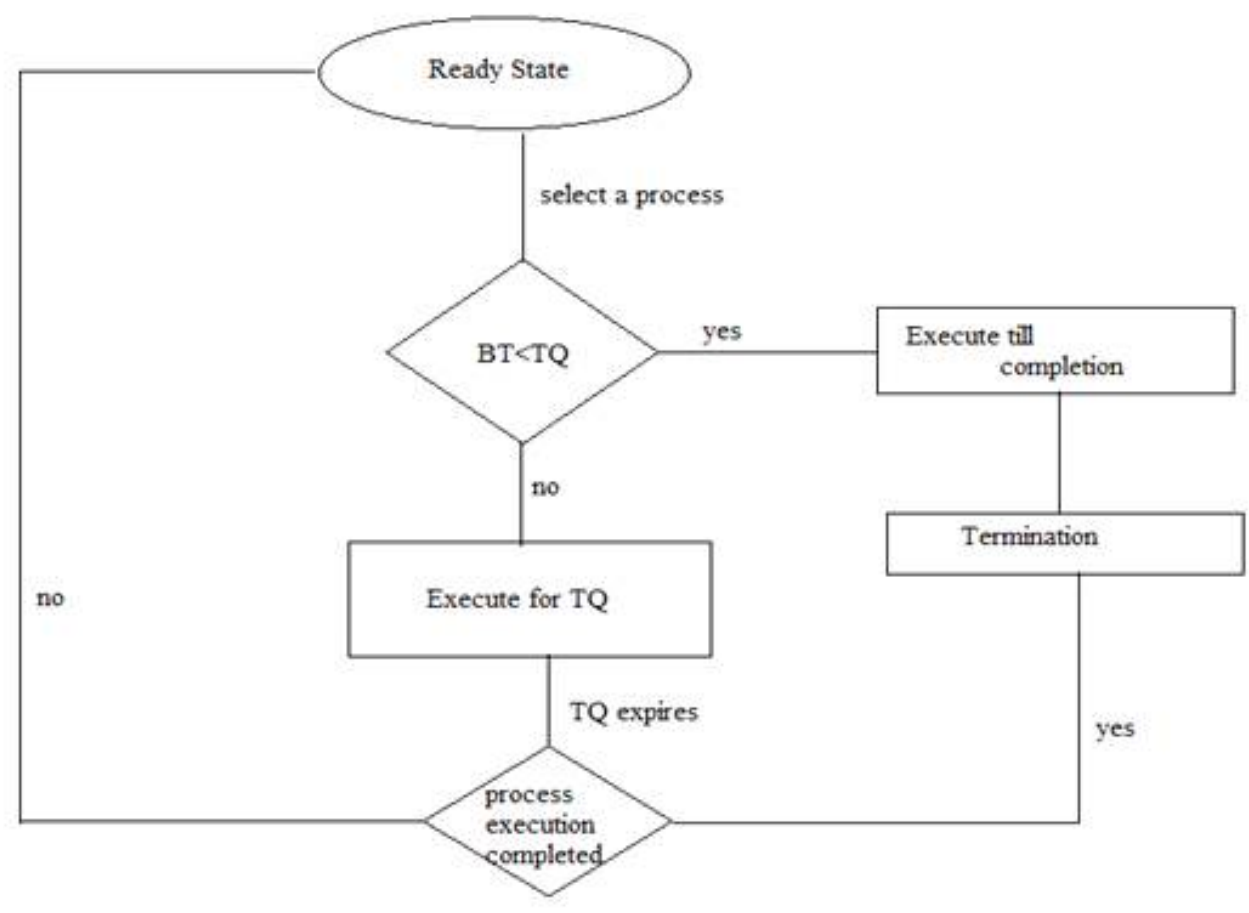

[Fig 1] Flow Chart 
We assume five processes arriving at time $=0$, with increasing burst time $(\mathrm{P} 1=4, \mathrm{P} 2=5, \mathrm{P} 3=2$, $\mathrm{P} 4=1, \mathrm{P} 5=6, \mathrm{P} 6=3$ )

\begin{tabular}{|c|c|c|}
\hline Processes & Arrival Time & Burst Time \\
\hline P1 & 0 & 5 \\
\hline P2 & 1 & 2 \\
\hline P3 & 2 & 1 \\
\hline P4 & 3 & 6 \\
\hline P5 & 4 & 3 \\
\hline P6 & 6 & 5 \\
\hline
\end{tabular}

[Fig. 2] Example for RR Algorithm

The Gantt chart is shown below:

\begin{tabular}{|c|c|c|c|c|c|c|c|c|c|c|c|}
\hline P1 & P2 & P3 & $\mathbf{P}_{1}$ & P4 & P5 & $\mathbf{P} 2$ & P6 & P5 & $\mathbf{P} 2$ & P6 & Ps \\
\hline
\end{tabular}

[Fig. 3] Gantt chart

Average Waiting Time $=8.04$

Average Turn Around Time= 11.16

Context Switching (if Time Quantum decreases CS increases and vice versa) $=7$

\subsection{Drawback}

At one extreme, if the time quantum is extremely giant, cause less response time and it's like FCFS. If the time quantum is extremely tiny this causes too several context switches and lowers the hardware potency

\subsection{Throttled Algorithm}


Throttled calculation is totally in sight of virtual machine. Here the client 1st demands the load balancer to check the privilege virtual machine that get thereto load effortlessly and play out the operations that is given by the customer. In this calculation the customer 1st demands the load balancer to find Associate in Nursing acceptable Virtual Machine to play out the desired operation. In the present work we tend to area unit pondering spherical Robin, Throttled calculation as an examination in light-weight of the reality that these calculations flow into the load systematically. Our attention is on enhancing the Throttled calculation which is accomplished basically. In this calculation, each VM is having a listing state and in this file state it having the state like sit while not moving state, occupied and accessible state. As indicated by the record express the load balancer, which goes concerning because the throttled calculation disseminates as per the VM file state.

\subsection{Min-Min Algorithm}

Min-Min start with the set of all unassigned tasks in the create span. This algorithm work in 2 phases. First, the minimum expected completion time for all the tasks is calculated. The completion time for all the tasks is calculated on all the machines. In the second phase, the task with the minimum expected completion time from make span is elect which tasks allotted to the corresponding resource. Then the task which is completed that is aloof from the create span and this method is recurrent till all tasks area unit completed.

\subsection{Max-Min Algorithm}

Max-Min start with the set of all unassigned tasks in the create span. This algorithm additionally works in 2 phases. First, the maximum expected completion time for all the tasks is calculated. The completion time for all the tasks is calculated on all the machines. In the second phase, the task with the maximum expected completion time from createspan is chosen which tasks allotted to the corresponding resource. Then the task which is completed that is aloof from the create span and this method is recurrent till all tasks area unit completed.

Shortest Job First algorithmic rule: As the name says whichever is that the shortest job within the population are going to be executed 1st. It is a non-pre-emptive scheduling algorithmic rule. SJF is simple and advantageous as a result of it produces less watch for the method compared to FCFS. 
Steps for Shortest Job First Algorithm (SJF)

Step 1: for job $=0$ to job $<$ main queue-size

if task $i+1$ length $<$ task $i$ length then

(a) add task $i+1$ in front of task $i$ in the queue

end if

Step 2: if main queue-size $=0$ then

(b) task i last in the main queue

end if

Step 3: end for

Drawback: When a job with larger burst time comes with highest priority it will not execute as it is a non-pre-emptive scheduling algorithm.

\subsection{Proposed Algorithm}

In our proposed formula, Modified spherical Robin formula. The number of processes is residing within the prepared queue, we assume their arrival time is assigned to some processes and burst times ar allotted to the computer hardware. The burst time and the number of processes ar thought-about as input. Now initial of all we tend to organize all processes in increasing order according to their given burst time and opt for changed time slice are going to be depends on the quantity of processes burst time. If number of processes ar varied then time slice can vary.

In this algorithm some variety of processes and their burst time ar given. And we have to be compelled to determine their context switch time their turnaround and their waiting time.

In modified spherical robin formula shortest job initial and spherical robin algorithms ar mixed up. So that this formula will take the benefits of each formula. And execute more expeditiously.

\section{Steps for Modified spherical Robin formula}

Step 1: first check the standing of prepared queue.

Step 2: change the standing of all the processes to prepared state.

Step 3: arrange all the processes in increasing order or type processes according to their burst time.

Step 4: check whether prepared queue is null or not. 
Step 5: if not then calculate modified time quantum

Time Quantum= No of Burst times/ No of processes.

Step 6: assign modified time quantum to each method.

Step 7: if burst time of method is smaller and equal to time quantum then process complete its execution.

Otherwise repeat cycle and give the time quantum to every method.

Step 8: repeat step 6 till all processes will not complete their execution.

\begin{tabular}{|c|c|c|}
\hline Processes & Arrival Time & Burst Time \\
\hline P1 & 0 & 4 \\
\hline P2 & 1 & 5 \\
\hline P3 & 2 & 2 \\
\hline P4 & 3 & 1 \\
\hline P5 & 4 & 6 \\
\hline P6 & 6 & 3 \\
\hline
\end{tabular}

[Fig. 4] Example for Modified RR

Quantum= AVG. of Burst time/No of processes

Time Quantum $=4$

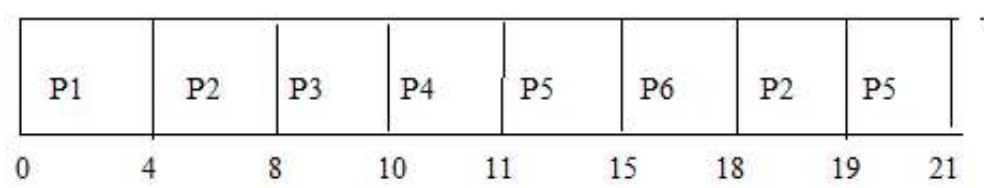

[Fig. 5] The Gantt chart

Average Waiting Time $=7.56$

Average Turnaround Time $=10.833$

Context Switching $=6$

\section{CONCLUSION}


Existing algorithm in cloud load balancing is not giving much efficient results like high waiting time, response time, context switching and throughput is low. But the efficient cloud load balancing should have the characteristics like low waiting time, response time, context switch and high throughput.

[Table 1] Comparison Table of RP and MBR

\begin{tabular}{|c|c|c|c|c|}
\hline Algorithm & WT & TAT & CS & Throughput \\
\hline RP & 8.04 & 11.16 & 7 & Low \\
\hline MBR & 7.56 & 10.833 & 6 & High \\
\hline
\end{tabular}

For that here it is represents a slight change in the round robin algorithm, called as modified round robin algorithm. This modified round robin algorithm is the combination of shortest job first(SJF) and round robin(RR). By calculating the modified round robin the parameters like high waiting time, response time are overcome. The modified round robin gives the better performance than round robin algorithm. The parameters like waiting time, average turnaround time and total number of context switches are reduced.

\section{References}

[1] S. Dave and P. Maheta, Utilizing Round Robin Concept for Load Balancing Algorithm at Virtual Machine Level in Cloud Environment, International Journal of Computer Applications, (2014), Vol.94, No.4, pp.23-29.

[2] P. Samal and P. Mishra, Analysis of variants in Round Robin Algorithms for load balancing in Cloud Computing, International Journal of Computer Science and Information Technologies, (2013), Vol.4, No.3, pp.416-419.

[3] P. Singh, P. Baaga, and S. Gupta, Assorted Load Balancing Algorithms in Cloud Computing: A Survey, International Journal of Computer Applications, (2016), Vol.143, No.7, pp.34-40.

[4] S. Supreeth and B. Shobha, Scheduling Virtual Machines for Load balancing in Cloud Computing Platform, International Journal of Science and Research, (2013), Vol.2, No.6, pp.437-441.

[5] P. M. Shameem and R. S. Shaji, A Methodological Survey on Load Balancing Techniques in Cloud Computing, Asian Journal of Information Technology, (2013), Vol.12, No.5, pp.160-169.

[6] A. Sirohi, A. Pratap, and M. Aggarwal, Improvised Round Robin (CPU) Scheduling Algorithm, International Journal of Computer Applications, (2014), Vol.99, No.18, pp.40-43. 
[7] S. K. Panda and S. K. Bhoi, An Effective Round Robin Algorithm using Min-Max Dispersion Measure, International Journal on Computer Science and Engineering, (2012), Vol.4, No.1, pp.45-53.

[8] S. M. Mostafa, S. Z. Rida, and S. H. Hamad, Finding Time Quantum of Round Robin CPU Scheduling Algorithm in General Computing Systems using Integer Programming, IJRRAS, (2010), Vol.5, No.1, pp.64-71.

[9] H. S. Behera, R. Mohanty, and D. Nayak, A New Proposed Dynamic Quantum with Re-Adjusted Round Robin Scheduling Algorithm and Its Performance Analysis, (2010), Vol.5, No.5, pp.10-15. 